\title{
Delirium associated with olanzapine therapy in a patient with Bipolar Affective Disorder: A Case Report.
}

\author{
Shrestha R1, Singh PM², Joshi N1, Dhonju G'
}

1.Junior Resident, Department of psychiatry, NMC, Kathmandu, Nepal 2.Associate Prof. Department of psychiatry, NMC, Kathmandu, Nepal.

E-mail *Corresponding author: Rajesh69411@hotmail.com

\begin{abstract}
A patient of Bipolar Affective Disorder (BPAD) developed delirium after initiation and increment in the dosage of olanzapine. After hospital admission, we started olanzapine in combination with lithium. Olanzapine was gradually increased to $30 \mathrm{mg} /$ day and lithium gradually increased to $1000 \mathrm{mg} /$ day with serum lithium level at $0.85 \mathrm{mmol} / \mathrm{L}$. After discontinuation of olanzapine, there was a complete resolution of delirium. We, hereby, report the case of delirium associated with olanzapine therapy.
\end{abstract}

Keywords: olanzapine, atypical antipsychotics, delirium

\section{INTRODUCTION:}

Delirium is characterized by an acute decline in both level of conscious and cognition with particular impairment in attention. The prevalence of delirium at hospital admission ranges from 14 to $24 \%$, and incidence of delirium arising during hospitalization ranges from 6 to $56 \%$. One of the predisposing factors for delirium is treatment with drugs with anticholinergic properties. Evidence supports the role of cholinergic deficiency in delirium involved in rapid eye movement (REM) sleep, disturbance in attention and memory. ${ }^{1}$ Bipolar Affective Disorder (BPAD) is characterized by episodes in which the patient's mood and activity levels are significantly disturbed, it consists of elevation or irritable of mood, increased energy and activity as seen in mania; and lowering of mood and decreased energy and activity as seen in depression. ${ }^{2}$ In this paper, a case of BPAD with delirium following use of olanzapine therapy is presented in which patient was in good general physical health prior to olanzepine therapy. The report of delirium due to interaction between lithium and haloperidol was reported in $1974 .{ }^{3}$ Several other case reports have presented delirium and extrapyramidal signs following use of lithium and antipsychotic drugs (e.g. chlorpromazine, trifluperazine, thioridazine etc). ${ }^{4}$ In the United Kingdom, a trial of high dose olanzapine (range 20-60 mg/day)showed moderate to marked clinical improvement in patients with clozapineresistant schizophrenia. ${ }^{5}$ Anecdotal case reports have also highlighted the benefit of olanzapine 
at $50 \mathrm{mg} /$ day in patient with refractory schizoaffective disorder. ${ }^{6}$

\section{CASE REPORT:}

A 44 years old male farmer, Hindu by religion, was suffering from bipolar affective disorder for since two years. He had an episode of mania which was treated with mood stabilizers and psychotropic medications. He was maintaining well after his last episode which was two years back and stopped all medications.

Now, he was brought by his family with complaints of irritability, over-talkativeness, over-familiarity, restlessness, over-religiousness, disturbed sleep, suspiciousness and overactivity since 45 days. There was no associated history of head trauma, fever, unconsciousness, seizures, psychoactive substance use and other precipitating factors. There was no significant history of medical or surgical illnesses. General physical examination showed that vitals were stable. Neurological, cardiovascular, respiratory and abdominal examination showed no significant abnormalities. The mental status examination presented a middle aged male, conscious, nonaggressive, sitting in bed, with poor hygiene, increased psychomotor activity. The speech was increased in volume and rate, reaction time was decreased, and spontaneous talk was present. Mood was predominantly elated. Thought was coherent but occasionally irrelevant, with grandeur delusion and delusion of persecution. No perceptual abnormalities were present. He was well oriented to time, place and person. Memory was intact, judgment was impaired, and insight was grade 1 . The patient was diagnosed with bipolar affective disorder current episode mania with psychotic symptoms. He was admitted in the psychiatry ward and baseline investigations were sent which included complete blood count, kidney and liver function test, random blood sugar, VDRL, HIV, and HBsAg. The investigations were all within normal limits. He was started on oral atypical antipsychotic olanzapine 10 $\mathrm{mg} /$ day, gradually increased to $30 \mathrm{mg} /$ day over a period of 24 days, and lithium gradually increased to $1000 \mathrm{mg} /$ day. After 2 days of increment of olanzapine to $30 \mathrm{mg} /$ day, On 26 th day of admission, patient became confused, did not recognized hospital staff, was disoriented to time, place and person, and had altered sleep- wake cycle, he woke in the middle of the night and started roaming in the ward and lied on other patient's bed, he could not recall the happenings on the next day. There was no history of vomiting, fever, nausea, loose stool, headache, abdominal pain, Ataxia, dizziness, tremors, slurred speech, nystagmus and muscle weakness. The diagnosis of delirium was made. All baseline investigations complete blood count, kidney function test, liver function test, random blood sugar along serum lithium level were sent. All medications were hold. The patient's condition improved, he was fully conscious and oriented to time, place, and person. The serum lithium level was 0.84 $\mathrm{mmol} / \mathrm{L}$ and the baseline investigations were within normal limits. Then aripriprazole was started and gradually increased to $30 \mathrm{mg} /$ day over the next 3 weeks and lithium was decreased. The patient showed progressive improvement on aripriprazole and lithium therapy.

\section{DISCUSSION:}

Our case was diagnosed as case of delirium as per ICD-10 criteria. $^{2}$ There were features like fluctuating consciousness, disorientation, impaired recent memory and disturbed sleep wake cycle. The higher dose of olanzapine at 30 $\mathrm{mg}$ per day precipitated delirium in our patient. The Naranjo probability scale indicates that olanzapine was probable cause of delirium. ${ }^{7}$ Delirium, in our case, resolved after olanzapine was stopped. Case reports had shown higher dose of olanzapine can be used in treatment refractory schizophrenia and BPAD with depression. ${ }^{6}$

There are past reports of olanzapine associated delirium that developed in an elderly male with BPAD. ${ }^{8}$ Conversely, there have been few reports of delirium associated with olanzapine. ${ }^{9}$ Most of these have been in patient with other risk factor for delirium like combination with lithium intoxication with olanzapine. ${ }^{10,11}$

The anticholinergic property of olanzapine might have contributed to the pathophysiology of delirium induced by olanzapine.12 One hypotheses postulated that decreased cholinergic activity may lead to delirium. ${ }^{12}$ Patient with delirium have high serum anticholinergic activity as compared with those without delirium. 


\section{CONCLUSION:}

Patient developing delirium with olanzapine therapy should be clinically evaluated and other causes of delirium should be ruled out. There should be discontinuation of olanzapine therapy and once the delirium resolves, treatment should be started with other antipsychotic drugs.

\section{REFERENCES:}

1. Sadock BJ, Sodock VA, Ruiz P, Kaplan and Shaddock's Comprehensive test book of psychiatry. 9th ed; Vol 1 Lippincott Williams and Williams; 2009.

2. WHO. The ICD-10 classification of mental and behavioral disorders: clinical descriptions and diagnostic guidelines. World Health Organization, Geneva. 2002.

3. Cohen WJ, Cohen NH, Lithium carbonate, haloperidol and irreversible brain damage. JAMA 1974; 230:1283-7.

4. Mani J, Tandel SV, Shah PU, Karnad DR. Prolonged neurological sequelae after combination treatment with lithium and antipsychotic drugs. J Neurol Neurosurg Psychiat 1996; 60:350-1.

5. Mountjoy CQ, Baldacchino AM, Stubbs JH. British experience with high dose olanzapine for treatment refractory schizophrenia .Am J Psychiatry.1999; 156:158-159.

6. Reich J. Use of high dose olanzapine for treatment refractory schizophrenia. Clin Neuropharmacol.2003; 26:58-61.

7. Naranjo CA, Busto $U$, Sellers EM, Sandor P, Ruiz I, Roberts EA, et al. A method for estimating the probability of adverse drug reactions. Clin Neurosci, 2001; 55:515-519.

8. Psychiatry Investig.2010 Jun; 7(2):153-154.

9. Samuels, Fang M. Olanzapine may cause delirium in geriatric patients: I clan Psychiatrc .2004; 65:582-83.

10. J Korean Med Sci 2005; 20:691-4.

11. Togluc, Erdugan E, Abay E. Delirium and extrapyramidal symptom due to lithium olanzapine combination therapy: a case report: J Korean Med Sci; 20:691-94.

12. Richelson E. Preclinical pharmacology of neuroleptics - focus on new generation compounds. I clin Psychaitry.1996; 57 (suppl 11):4-11. 